\title{
Hemispheric asymmetry in discriminating faces differing for featural or configural (second-order relations) aspects
}

\author{
Zaira Cattaneo $\cdot$ Chiara Renzi • Silvia Bona • \\ Lotfi B. Merabet • Claus-Christian Carbon • \\ Tomaso Vecchi
}

(C) Psychonomic Society, Inc. 2013

\begin{abstract}
The human capacity to discriminate among different faces relies on distinct parallel subprocesses, based either on the analysis of configural aspects or on the sequential analysis of the single elements of a face. A particular type of configural processing consists of considering whether two faces differ in terms of internal spacing among their features, referred to as second-order relations processing. Findings from electrophysiological, neuroimaging, and lesion studies suggest that, overall, configural processes rely more on the right hemisphere, whereas analysis of single features would involve more the left. However, results are not always consistent, and behavioral evidence for a right-hemisphere specialization in second-order relations processing is lacking. Here, we used divided visual field presentation to investigate the possible different contributions of the two hemispheres to face discrimination based on relational versus featural processing.
\end{abstract}

\section{Z. Cattaneo}

Department of Psychology, University of Milano-Bicocca, Milano, Italy

Z. Cattaneo $(\varangle) \cdot$ C. Renzi $\cdot$ T. Vecchi

Brain Connectivity Center, National Neurological Institute C.

Mondino, Pavia, Italy

e-mail: zaira.cattaneo@unimib.it

\section{S. Bona}

Brain Research Unit, O.V. Lounasmaa Laboratory, School of Science, Aalto University, Espoo, Finland

L. B. Merabet

Department of Ophthalmology, Massachusetts Eye and Ear Infirmary, Harvard Medical School, Boston, USA

\section{C.-C. Carbon}

Department of General Psychology and Methodology, University of Bamberg, Bamberg, Germany

T. Vecchi

Department of Brain and Behavioral Sciences, University of Pavia, Pavia, Italy
Our data indicate a right-hemispheric specialization in relational processing of upright (but not inverted) faces. Furthermore, we provide evidence regarding the involvement of both the right and left hemispheres in the processing of faces differing for inner features, suggesting that both analytical and configural modes of processing are at play.

Keywords Configural $\cdot$ Featural $\cdot$ Face processing . Lateralization $\cdot$ Divided visual field $\cdot$ Jane faces task

\section{Introduction}

The ability that humans have to quickly detect faces among other objects and to discriminate among the multitude of different faces encountered in everyday life depends on several types of processing (for reviews, see Maurer, Le Grand, \& Mondloch, 2002; Tsao \& Livingstone, 2008). In particular, first-order information (i.e., the basic attributes present in each face, such as two eyes, a nose, a mouth) is used for holistic processing of a face, allowing one to quickly discriminate a face from a nonface stimulus (Maurer et al., 2002). Furthermore, to discriminate among different faces, individuals rely on both (1) the analysis of single features within the face (such as the shape, color, or texture of the eyes or the nose), a type of encoding known as featural processing, and (2) processing of second-order configuration of these features (e.g., the distance between the eyes or between the eyes and the mouth), or relational processing (Maurer et al., 2002). Converging evidence suggests that featural and relational types of face processing rely on different although strongly associated mechanisms (Freire, Lee, \& Symons, 2000; Leder \& Carbon, 2006; Lobmaier, Bolte, Mast, \& Dobel, 2010; Mondloch, Le Grand, \& Maurer, 2002; Rotshtein, Geng, Driver, \& Dolan, 2007).

In fact, featural and relational processes (and also holistic processing of faces) are also likely to be mediated by different neural networks, involving the two hemispheres differently 
(e.g., Maurer et al., 2007; Mercure, Dick, \& Johnson, 2008; Pitcher, Walsh, Yovel, \& Duchaine, 2007; Rossion et al., 2000; Scott \& Nelson, 2006). In particular, holistic processing and processing of second-order relations of faces are likely to be mediated mainly by the right hemisphere (RH), whereas analysis of the single features is likely to rely mainly on the left hemisphere (LH). Results are not entirely consistent however. For instance, using the divided visual field (DVF) methodology (in which stimuli are selectively presented in the left visual field (LVF) or right visual field (RVF), thus preferentially activating the contralateral hemisphere), Hillger and Koenig (1991) reported an RVF/LH advantage in a same-different judgment task for faces in which faces differed for a single feature (the same result was also reported by Parkin \& Williamson, 1987). However, when faces differed for more than one feature, an LVF/RH advantage was reported (Hillger \& Koenig, 1991). Other studies using the DVF paradigm to assess hemispheric specialization for holistic processing of faces have generally reported an LVF/RH advantage (Parkin \& Williamson, 1987; Ramon \& Rossion, 2012). Although processing of secondorder relations of faces has not been investigated so far through a DVF methodology, neuroimaging and electrophysiological evidence suggests that this type of processing taps more into the RH's resources (see Maurer et al., 2007; Scott \& Nelson, 2006). In particular, fMRI evidence suggests a prevalent left-frontalhemisphere activation associated with featural processing and a prevalent right fronto-parietal activation associated with processing of second-order relations of faces (Maurer et al., 2007). ERP evidence also indicates a major contribution of the LH to featural processing and of the RH to relational processing of faces (Scott \& Nelson, 2006). Nonetheless, a brain stimulation study (Pitcher et al., 2007) showed that a face-specific region in the $\mathrm{RH}$, the occipital face area, plays a critical role in processing face parts, but not in processing the spacing between these parts. Furthermore, other neuroimaging studies failed to report a different activation in face-selective regions - such as the fusiform face area - depending on detection of featural versus relational changes (Yovel \& Kanwisher, 2004; see also Maurer et al., 2007; but see Rotshtein et al., 2007).

In this study, we used a lateralized stimulus presentation to investigate possible hemispheric differences in judging the identity of two consecutively presented faces differing in either featural or (second-order) configural aspects. This is particularly interesting since processing of second-order relations of faces has not yet been investigated using a DVF methodology. The use of this method is also relevant in light of controversial results previously reported in the literature when discrimination for faces differing for facial features was investigated (Hillger \& Koenig, 1991; Maurer et al., 2007; Pitcher et al., 2007). For the purpose of our investigation, we used the so-called Jane faces task (Maurer et al., 2007; Mondloch et al., 2002), in which the same face (Jane) is modified to obtain eight different versions ("Jane's sisters"): four differing by single elements (the shape of eyes and the mouth; featural set) and four differing by spacing among the same facial elements (relational set). Notably, each set (featural and relational) was also presented with faces in an upside-down orientation. Although inversion impairs face discrimination overall, it seems that with inverted faces, a configural mode of processing cannot be efficiently applied (e.g., Le Grand, Mondloch, Maurer, \& Brent, 2001; Mondloch et al., 2002; Robbins, Nishimura, Mondloch, Lewis, \& Maurer, 2010). Accordingly, the inversion manipulation in our DVF experiment allows shedding further light on possible hemispheric asymmetry in the different mechanisms underlying face processing.

\section{Method}

\section{Participants}

Twenty students ( 5 males; mean age $=23.2$ years, $S D=1.60$; range: 19-27 years), all right-handed (Oldfield, 1971), took part in the experiment. All had normal or corrected-to-normal vision. Written informed consent was obtained from all participants, and the study was approved by the local ethical review committee.

\section{Materials and procedure}

Participants sat $57 \mathrm{~cm}$ from a 17 -in. computer monitor $(1,440 \times$ 900; refresh rate: $60 \mathrm{~Hz}$ ). A chinrest was used to ensure that the head was aligned with the middle of the screen and that the distance from the screen was kept constant. E-Prime 2 (Psychology Software Tools, Pittsburgh, PA) was used for stimuli presentation and data collection.

Face stimuli (see Fig. 1a) subtended $5^{\circ}$ of visual angle in width and $8^{\circ}$ of visual angle in height and consisted of grayscale photographs (image resolution: $72 \times 72 \mathrm{dpi}$ ) of a single Caucasian young female face (called "Jane") and in its featural and relational variants ("Jane's sisters") (see Mondloch et al., 2002, for details). In particular, four featural-different variants (equally different from the original Jane) were created by replacing the original Jane face's eyes and mouth with the features of the same length from different females. Four relational-different variants (equally different from the original Jane) were created by moving the eyes of the original Jane up or down, closer together or farther apart, and the mouth up or down (more specifically, Jane's eyes were moved up or down by $0.95 S D$ and closer together or farther apart by $2.60 S D$, while Jane's mouth was moved up or down $1.06 S D$; see Mondloch et al., 2002, for details). The featural and relational sets were presented in separate blocks to allow time for each style of processing to emerge, but participants were not explicitly informed about the distinctions (see Maurer et al., 2007). Each set was presented both with faces appearing in the standard upright orientation and with faces appearing upside-down 
(inverted orientation). The inverted set was always presented after the upright set.

A DVF paradigm was used (following criteria recommended by Bourne, 2006). The timeline of an experimental trial is presented in Fig. 1b. Each trial started with a central fixation cross (duration: 1,000 ms), followed by the probe face, which appeared in the middle of the screen for $150 \mathrm{~ms}$ in the featural set (the same duration was also used in the DVF study by Hillger \& Koenig, 1991, with faces differing in single or multiple features) and for $180 \mathrm{~ms}$ in the relational set. ${ }^{1}$ The central fixation cross was then presented. After $500 \mathrm{~ms}$ since the onset of the fixation cross, the target face was presented for $150 \mathrm{~ms}$ in the featural set and for $180 \mathrm{~ms}$ in the relational set, the inner border of the laterally presented face being located either $3^{\circ}$ to the left or $3^{\circ}$ to the right of the fixation cross (see Bourne, 2006). The central fixation cross remained visible until the participant responded. The following trial was initiated by participants' response. Participants responded with left/right keypresses (response key assignment was counterbalanced across participants), using their left and right index fingers, and were instructed to maintain fixation on the central fixation cross while performing the task. In each block (featural upright, featural inverted, relational upright, relational inverted), all the possible pair-combinations of Jane and its four variants were presented in random order, with each face appearing an equal number of times in the LVF and in the RVF. Each block consisted of 160 trials (80 different trials and 80 same trials). Each face appeared an equal number of times as probe and target face.

Before the experiment, a short slide presentation was shown to explain the task. The difference or identity between stimuli was emphasized, but no explicit cues were provided on the type of changes that could occur. Furthermore, prior to the task, short practice blocks for each set and orientation were performed in order to familiarize participants with the task. Response speed was encouraged, in addition to accuracy. The importance of maintaining fixation in the center of the screen was stressed throughout the task. The whole experiment lasted approximately $1 \mathrm{~h}$.

\section{Results}

Participants' mean accuracy in the different experimental conditions is reported in Table 1 . Trials $(1.46 \%$ of the total trials number) in which individual response latencies were beyond 3 standard deviations with respect to each

\footnotetext{
${ }^{1}$ A longer presentation duration was chosen for the relational set to avoid potential floor effects with inverted faces (note that accuracy in the inverted relational set was slightly above chance, but we preferred not to further increase duration in order to avoid potential confounds of eye movements; see Bourne, 2006, p. 381: "it is recommended that stimulus presentation is limited to a maximum exposure of $180 \mathrm{~ms}$, with exposure ideally limited to $150 \mathrm{~ms}$ if the task is a simple one").
}

participant's mean performance in each experimental block were excluded from the analyses. Performance was considered in terms of detection sensitivity $\left(d^{\prime}\right.$; see MacMillan \& Creelman, 1991), in light of previous evidence (cf. Hillger \& Koenig, 1991) suggesting that hemispheric asymmetry in processing faces may be different depending on type of considered trial (same and different trials). Moreover, we also investigated the existence of possible individual response biases ( $c$; MacMillan \& Creelman, 1991) and whether they were affected by VF and face orientation. Negative $c$ values indicate that the number of false alarms exceeds the number of misses (i.e., participants tend to respond different more often than they tend to respond same). Positive $c$ values indicate a more cautious response criterion, with the number of misses being higher than the number of false alarms (i.e., participants tend to respond same more often than they tend to respond different). Repeated measures ANOVAs with face orientation (upright vs. inverted) and visual field (LVF vs. RVF) as withinsubjects variables were carried on $d^{\prime}, c$, and mean correct RT for the featural and the relational set. Performance in the two sets was analyzed separately due to the different presentation times of face stimuli in the two conditions (see above).

\section{Relational set}

Mean $d^{\prime}$ and mean RT as a function of VF and experimental condition are reported in Fig. 2. The ANOVA on $d^{\prime}$ reveled a significant main effect of orientation, $F(1,19)=56.40, p<.001$, $\eta_{\mathrm{p}}{ }^{2}=.75$, with upright faces being discriminated better than upside-down faces. Detection of differences was significantly higher in the LVF than in the RVF, $F(1,19)=6.87, p=.017$, $\eta_{\mathrm{p}}{ }^{2}=.27$, but the effect depended on face orientation, as suggested by the significant $\mathrm{VF} \times$ orientation interaction, $F(1,19)=$ $4.51, p=.047, \eta_{\mathrm{p}}^{2}=.19$. Post hoc comparisons indicated that face discrimination was more efficient in the LVF than in the RVF for upright faces, $t(19)=2.98, p=.008$. Conversely, no differences related to VF were, overall, observed for faces presented upside-down, $t(19)<1, p=.530$.

Analyses on the response bias $c$ showed a significant effect of VF, $F(1,19)=13.49, p=.002, \eta_{\mathrm{p}}{ }^{2}=.41$ : Although the number of false alarms exceeded number of misses in both VFs (reflecting an overall "liberal" response criterion), participants were nonetheless significantly more conservative in their judgments when faces appeared in the LVF than when they appeared in the RVF. Response criterion tended also to be more liberal for inverted than for upright faces, although not to a significant extent (main effect of orientation: $p=.13$ ). The orientation $\times$ VF interaction $(p=.58)$ was not significant.

Analyses on mean correct RT showed no significant main effect of either orientation $(p=.09$, a trend being present indicating faster responses for inverted faces and likely reflecting a speed-accuracy trade-off) or VF $(p=.43)$. The $\mathrm{VF} \times$ orientation interaction was not significant $(p=.93)$. 
Featural set

In Fig. $3, d^{\prime}$ and mean correct RT for the featural set are reported. The analysis on $d^{\prime}$ revealed a significant main effect of orientation, $F(1,19)=69.51, p<.001, \eta_{\mathrm{p}}{ }^{2}=.79$, indicating better discrimination of upright faces than of upsidedown faces. The main effect of VF $(p=.76)$ was not significant. The VF $\times$ orientation interaction failed to reach significance $(p=.17)$. Analyses on the response bias $c$ revealed a significant main effect of both orientation, $F(1,19)=6.58$,

\section{a}
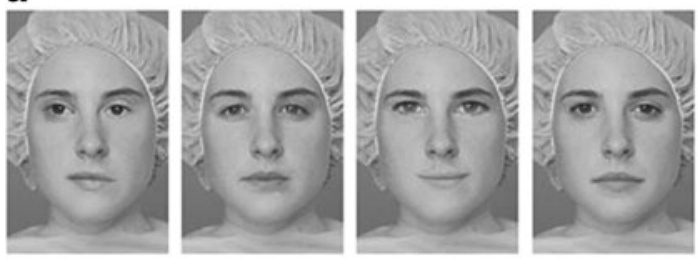

Jane's sisters - Featural set
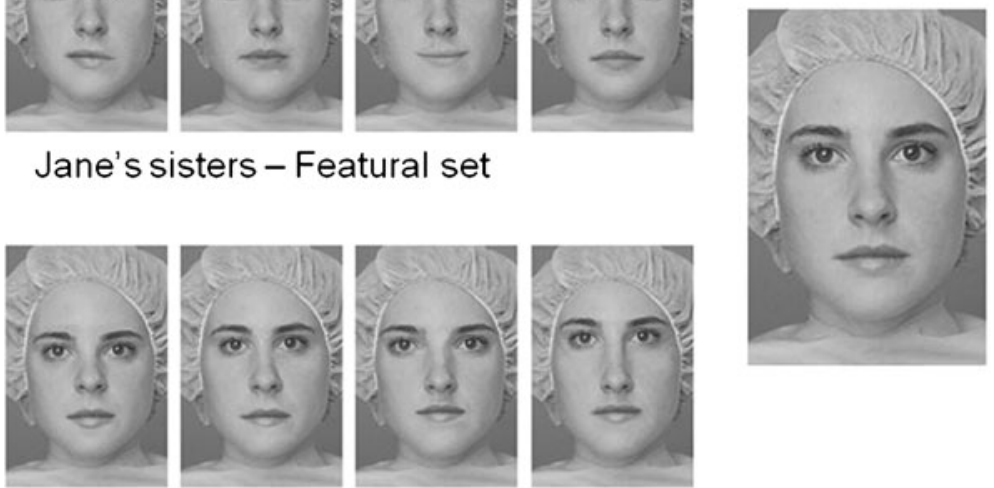

Jane

Jane's sisters - Relational set

\section{b}

\section{Fixation}

$1000 \mathrm{~ms}$

First face

$150 \mathrm{~ms}$ (featural set) or $180 \mathrm{~ms}$ (relational set)

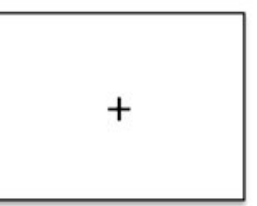

Fixation

$500 \mathrm{~ms}$

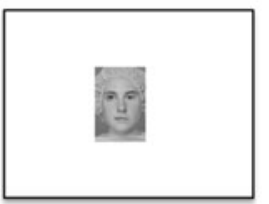

Second face

$150 \mathrm{~ms}$ (featural set) or $180 \mathrm{~ms}$ (relational set)

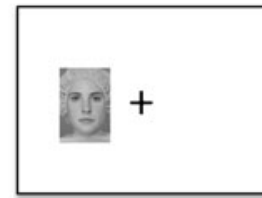

$+$

Blank screen

Until response

Fig. 1 a The Jane original face and her "sisters" differing for changes in single features or for the spacing (relational changes) among the face's elements. b The timeline of an experimental trial. Participants had to indicate whether the two faces were identical or not and were instructed to be both accurate and fast 
Table 1 Participants' mean percentage accuracy $(S D)$ for the relational and the featural set with upright and inverted faces

\begin{tabular}{llllll}
\hline & LVF & & & \multicolumn{2}{l}{ RVF } \\
\cline { 2 - 3 } \cline { 5 - 6 } \cline { 5 - 6 } & Upright & Inverted & & Upright & Inverted \\
\hline Relational set & $69.5(8.9)$ & $55.1(7.2)$ & & $64.9(9.8)$ & $54.0(8.1)$ \\
Featural set & $78.0(7.2)$ & $62.5(8.3)$ & & $74.7(10.3)$ & $64.9(10.9)$ \\
\hline
\end{tabular}

Note. LVF, left visual field; RVF, right visual field

$p=.019, \eta_{\mathrm{p}}{ }^{2}=.26$, and VF, $F(1,19)=6.68, p=.018, \eta_{\mathrm{p}}{ }^{2}$ $=.27$ : Participants showed overall a tendency toward responding different more often than toward responding same; this bias, though, was more evident in the RVF than in the LVF and was greater for inverted than for upright faces. The $\mathrm{VF} \times$ orientation interaction was not significant $(p=.73)$.

Analyses on mean correct RT showed only a significant effect of orientation, $F(1,19)=5.84, p=.02, \eta_{\mathrm{p}}{ }^{2}=.24$, with participants giving faster responses with inverted than with upright faces (again likely reflecting speed-accuracy tradeoff). Neither the main effect of VF ( $p=.73)$ nor the VF $\times$ orientation interaction $(p=.61)$ reached significance.
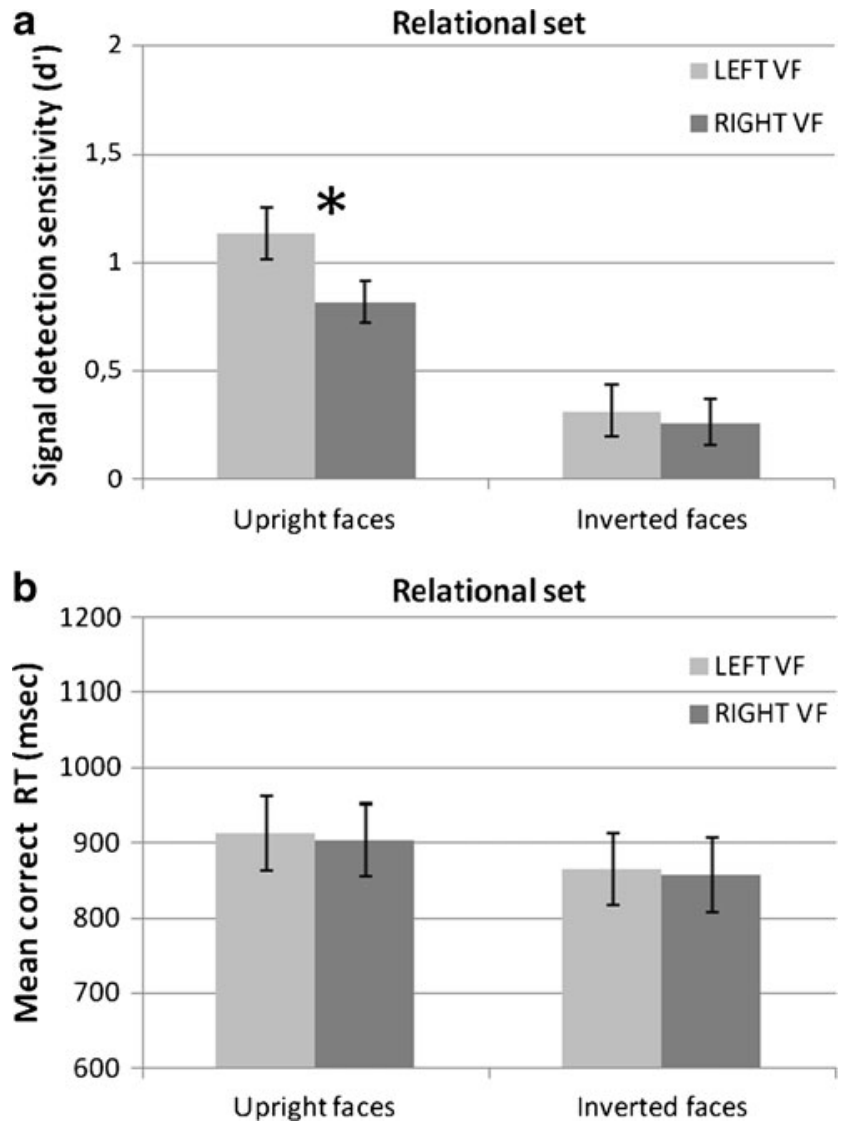

Fig. 2 Participants' a mean detection sensitivity $\left(d^{\prime}\right)$ and $\mathbf{b}$ response latencies (RTs, in milliseconds) for correct responses in the relational set of the Jane faces task for upright and inverted faces as function of visual field (VF). Error bars represent $\pm 1 S E M$. Asterisks indicate a significant difference between the two VFs

\section{Discussion}

In this study, we used a DVF paradigm to investigate possible hemispheric asymmetries in discriminating among faces differing in either second-order configural changes or facial features presented in either a standard upright or inverted (i.e., upside-down) orientation.

To our knowledge, face discrimination differing in second-order configural changes has not yet been investigated using a DVF methodology. Our findings showed an overall LVF/RH advantage in discriminating upright faces differing for spacing among otherwise identical features (relational set). Conversely, discrimination of faces differing for inner features (featural set) was not significantly affected by the VF manipulation. In both VFs and for both types of processing (featural and configural), performance was higher for upright than for inverted faces.

The LVF/RH advantage found for relational processing of upright faces has been corroborated by previous neuroimaging and ERP evidence indicating a $\mathrm{RH}$ specialization in discriminating faces on the basis of differences in their second-order relations (Maurer et al., 2007; Scott \& Nelson,
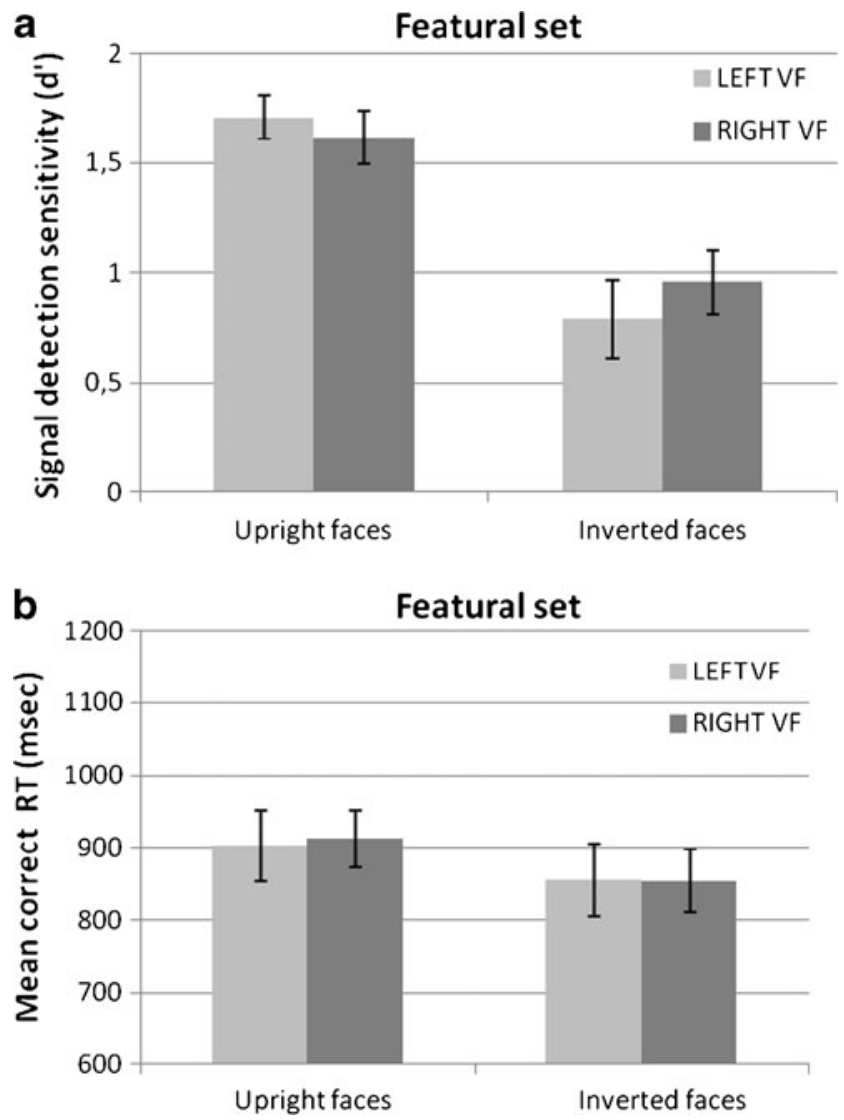

Fig. 3 Participants' a mean $d^{\prime}$ and $\mathbf{b}$ response times (RTs, in milliseconds) for correct responses in the featural set of the Jane faces task for upright and inverted faces as a function of visual field (VF). Error bars represent $\pm 1 S E M$ 
2006). Conversely, no VF advantage was observed for inverted faces. The absence of a LVF/RH advantage with inverted faces we reported here is consistent with previous evidence indicating that inversion is specifically detrimental for configural modes of face processing (cf. Leder \& Carbon, 2006; McKone \& Yovel, 2009; Rakover, 2012). In fact, prior findings collected with cataract reversal patients using the same face set as that used here (Le Grand et al., 2001; Robbins et al., 2010) showed that inversion particularly disrupts a configural mode of processing that seems to be specific for human faces presented in their standard upright orientation; a process that is not employed for other faces such as animals (e.g., monkeys) or objects (e.g., houses). When the configural mode of encoding is prevented by inversion, the role of the RH becomes less prominent, in agreement with our results. Nonetheless, it is worth mentioning that relational processing was near chance levels with the inverted face stimuli (and also replicating previous results; see Monldoch et al., 2002). Therefore, we cannot exclude the possibility that floor effects masked the emergence of hemispheric differences in this condition.

Notably, we did not find evidence for a clear hemispheric asymmetry when considering discrimination of faces differing for inner features (i.e., mouth and eyes). Previous fMRI and ERP evidence has suggested a more prominent role of the LH, as compared with the RH, in encoding featural aspects of faces (Maurer et al., 2007; Scott \& Nelson, 2006). However, a LH preference for featural processing versus second-order relational processing has not been replicated in other fMRI and ERP studies (e.g., Mercure et al., 2008; Rotshtein et al., 2007). In considering our findings in the featural set, it is important to note that changing featural elements of a face inherently implies also a change in the resulting face configuration. Hence, configural processing is also involved in discriminating upright faces differing in inner features. In this respect, the results obtained by Hillger and Koenig (1991) are critical: Using a DVF paradigm, the authors reported a LVF/RH advantage for upright faces differing for multiple features (i.e., changes simultaneously affecting the eyes, the nose, and the mouth). However, when faces differed for only one facial feature, a more complex pattern emerged: When faces were identical (same trials), a LVF/RH advantage was found (but this advantage disappeared when faces were presented upside-down in a further experiment); when faces differed (different trials), a RVF/LH advantage emerged (that was still evident when faces were presented upside-down). According to Hillger and Koenig, these findings provide evidence for the existence of a face-specific holistic process in discriminating upright faces that would be mediated mainly by the RH and that would be disrupted by inversion and for the existence of a more general (not face-specific) visual analytic process responsible for detection of single local changes mediated mainly by the LH and not affected by inversion. The extent to which configural and analytical processes are at play likely depends on the number of features that simultaneously change and on their salience (see Hillger \& Koenig, 1991). In our featural set, faces differed for the mouth and the eye features simultaneously. Our situation was thus intermediate between the singlefeature and the three-feature changes used by Hillger and Koenig. Our data seem to indicate that with upright faces, a configural mode of processing was playing a major role (as is shown in Fig. 3a, a trend was present toward an advantage for $\mathrm{LVF} / \mathrm{RH}$ ). When this configural processing was blocked by inversion, participants could still rely on analytical mode of processing as reflected by a slight advantage (see Fig. 3a) for $\mathrm{RVF} / \mathrm{LH}$ presentation (with this analytical mode of processing being of no help in case of spacing changes; see above).

One may object that the selective VF effects we reported in the relational set depended on differences in the level of difficulty between the featural and the relational task (the latter being more difficult), rather than tapping into two different encoding mechanisms. In this view, since the RH is specialized for face processing, its greater involvement with upright faces in the relational set might have depended on the relational task requiring more face-processing resources overall, rather than more relational processing per se. However, this possibility seems unlikely. In fact, if task difficulty was the main factor mediating our effects, a LVF/RH advantage should have emerged also with inverted faces in the featural set, given the comparable level of accuracy in this condition and in the upright condition of the relational set.

The VF effects reported in our experiment did not emerge when considering reaction times. Accordingly, several previous studies employing a sequential same-different face-matching task investigating featural versus spacing processing have selectively reported effects of experimental manipulations on accuracy measures (e.g., Keyes, 2012; Lobmaier et al., 2010; Pitcher et al., 2007; Rakover, 2012; Yovel \& Kanwisher, 2008), suggesting that reaction times may be a less sensitive measure than accuracy measures in these kinds of tasks.

In our analyses, we also considered possible effects due to preexisting response bias (i.e., individual a priori tendency to respond different or same; see MacMillan \& Creelman, 1991). Analyses showed that our participants tended to be overall more liberal than conservative, tending to respond different more often than same (i.e., the number of false alarms was greater than the number of misses). However, the different bias was significantly more evident for faces appearing in the RVF than for those appearing in the LVF in both sets and more evident overall with upright than with inverted faces (although significantly so only in the featural set). Overall, these results indicate that when the level of uncertainty was greater, the a priori response bias became stronger.

In sum, our results provide a straightforward behavioral demonstration of a right-hemispheric specialization in relational processing of upright faces, extending previous evidence obtained with fMRI and ERPs methodologies (e.g., 
Maurer et al., 2007; Scott \& Nelson, 2006) and adding to previous behavioral data obtained using DVF paradigms testing featural versus holistic (but not specifically relationalbased) processing of faces. Our data also provide evidence regarding the involvement of both $\mathrm{RH}$ and $\mathrm{LH}$ resources in the processing of faces differing for inner features, suggesting that both analytical and configural modes of processing are at play.

Acknowledgments The authors would like to thank Cathy Mondloch and her colleagues for providing the original Jane faces stimuli used by Mondloch et al. (2002), Susanna Schiavi for helping in data collection, and Carlo Toneatto for technical support. This work was supported by Vigoni/DAAD grant (years 2012-2013) to CCC and ZC.

\section{References}

Bourne, V. (2006). The divided visual field paradigm: Methodological considerations. Laterality, 11(4), 373-393.

Freire, A., Lee, K., \& Symons, L. A. (2000). The face-inversion effect as a deficit in the encoding of configural information: Direct evidence. Perception, 29(2), 159-170.

Hillger, L. A., \& Koenig, O. (1991). Separable mechanisms in face processing: Evidence from hemispheric specialization. Journal of Cognitive Neuroscience, 3, 42-58.

Keyes, H. (2012). Categorical perception effects for facial identity in robustly represented familiar and self-faces: The role of configural and featural information. The Quarterly Journal of Experimental Psychology, 65(4), 760-772.

Le Grand, R., Mondloch, C. J., Maurer, D., \& Brent, H. P. (2001). Neuroperception. Early visual experience and face processing. Nature, 410(6831), 890

Leder, H., \& Carbon, C. C. (2006). Face-specific configural processing of relational information. British Journal of Psychology, 97, 19-29.

Lobmaier, J. S., Bolte, J., Mast, F. W., \& Dobel, C. (2010). Configural and featural processing in humans with congenital prosopagnosia. Advances in Cognitive Psychology, 6, 23-34.

Macmillan, N. A., \& Creelman, C. D. (1991). Detection theory: A user's guide. New York: Cambridge University Press.

Maurer, D., Le Grand, R., \& Mondloch, C. (2002). The many faces of configural processing. Trends in Cognitive Sciences, 6, 255-260.

Maurer, D., O'Craven, K., Le Grand, R., Mondloch, C., Springer, M., Lewis, T., \& Grady, C. (2007). Neural correlates of processing facial identity based on features versus their spacing. Neuropsychologia, 45, 1438-1451.

McKone, E., \& Yovel, G. (2009). Why does picture-plane inversion sometimes dissociate perception of features and spacing in faces, and sometimes not? Toward a new theory of holistic processing. Psychonomic Bulletin \& Review, 16(5), 778-797.

Mercure, E., Dick, F., \& Johnson, M. H. (2008). Featural and configural face processing differentially modulate ERP components. Brain Research, 1239, 162-170.

Mondloch, C. J., Le Grand, R., \& Maurer, D. (2002). Configural face processing develops more slowly than featural face processing. Perception, 31, 553-566.

Oldfield, R. C. (1971). The assessment and analysis of handedness: The Edinburgh inventory. Neuropsychologia, 9(1), 97-113.

Parkin, A. J., \& Williamson, P. (1987). Cerebral lateralisation at different stages of facial processing. Cortex, 23(1), 99-110.

Pitcher, D., Walsh, V., Yovel, G., \& Duchaine, B. (2007). TMS evidence for the involvement of the right occipital face area in the early face processing. Current Biology, 17(18), 1568-1573.

Rakover, S. S. (2012). A feature-inversion effect: Can an isolated feature show behaviour like the face-inversion effect? Psychonomic Bulletin \& Review, 19(4), 617-624.

Ramon, M., \& Rossion, B. (2012). Hemisphere-dependent holistic processing of familiar faces. Brain and Cognition, 78(1), 7-13.

Robbins, R. A., Nishimura, M., Mondloch, C. J., Lewis, T. L., \& Maurer, D. (2010). Deficits in sensitivity to spacing after early visual deprivation in humans: A comparison of human faces, monkey faces, and houses. Developmental Psychobiology, 52, 775-781.

Rossion, B., Dricot, L., Devolder, A., Bodart, J. M., Crommelinck, M., De Gelber, B., \& Zoontjes, R. (2000). Hemispheric asymmetries for whole-based and part-based face processing in the human fusiform gyrus. Journal of Cognitive Neuroscience, 12(5), 793-802.

Rotshtein, P., Geng, J. C. A., Driver, J., \& Doland, R. J. (2007). Role of features and second-order spatial relations in face discrimination, face recognition, and individual face skills: Behavioral and functional magnetic resonance imaging data. Journal of Cognitive Neuroscience, 19(9), 1435-1452.

Scott, L. S., \& Nelson, C. A. (2006). Featural and configural face processing in Aadults and infants: A behavioral and electrophysiological investigation. Perception, 35(8), 1107-1128.

Tsao, D. Y., \& Livingstone, M. S. (2008). Mechanisms of face perception. Annual Review of Neuroscience, 31, 411-437.

Yovel, G., \& Kanwisher, N. (2004). Face perception: Domain specific, not process specific. Neuron, 44, 889-898.

Yovel, G., \& Kanwisher, N. (2008). The representations of spacing and part-based information are associated for upright faces but dissociated for objects: Evidence from individual differences. Psychonomic Bulletin \& Review, 15(5), 933-939. 